\title{
Effects of target pre-heating and expansion on terahertz radiation production from intense laser-solid interactions
}

\author{
X.H. Yuan ${ }^{1,2}$, Y. Fang ${ }^{1}$, D.C. Carroll ${ }^{3,4}$, D.A. MacLellan ${ }^{3}$, F. Du ${ }^{2}$, N. Booth ${ }^{4}$, M. Burza ${ }^{5}$, M. Chen ${ }^{1}$, \\ R.J. Gray ${ }^{3}$, Y.F. Jin ${ }^{1}$, Y.T. Li ${ }^{2}$, Y. Liu ${ }^{1}$, D. Neely ${ }^{4}$, H. Powell ${ }^{3}$, G. Scott ${ }^{3,4}$, C.-G. Wahlström ${ }^{5}$, J. Zhang ${ }^{1,2}$, \\ P. McKenna ${ }^{3}$, and Z.M. Sheng ${ }^{1,3}$ \\ ${ }^{1}$ Key Laboratory for Laser Plasmas (Ministry of Education) and Department of Physics and Astronomy, Shanghai Jiao Tong University, \\ Shanghai 200240, China \\ ${ }^{2}$ Beijing National Laboratory of Condensed Matter Physics, Institute of Physics, Chinese Academy of Sciences, Beijing 100190, China \\ ${ }^{3}$ SUPA Department of Physics, University of Strathclyde, Glasgow G4 ONG, UK \\ ${ }^{4}$ Central Laser Facility, Rutherford Appleton Laboratory, Oxfordshire OX11 OQX, UK \\ ${ }^{5}$ Department of Physics, Lund University, S-22100 Lund, Sweden \\ (Received 8 February 2014; revised 8 February 2014; accepted 19 February 2014)
}

\begin{abstract}
The first experimental measurements of intense $\left(\sim 7 \times 10^{19} \mathrm{~W} \mathrm{~cm}^{-2}\right)$ laser-driven terahertz (THz) radiation from a solid target which is preheated by an intense pulse of laser-accelerated protons is reported. The total energy of the THz radiation is found to decrease by approximately a factor of 2 compared to a cold target reference. This is attributed to an increase in the scale length of the preformed plasma, driven by proton heating, at the front surface of the target, where the $\mathrm{THz}$ radiation is generated. The results show the importance of controlling the preplasma scale length for $\mathrm{THz}$ production.
\end{abstract}

Keywords: target heating; terahertz radiation

\section{Introduction}

Investigation of terahertz $(\mathrm{THz})$ radiation from intense laserproduced plasmas is important both from the perspective of exploring new fundamental physics and because of the potential to develop compact sources for application. Highpower laser-produced plasma provides a unique environment for exploring matter under extreme temperature and density conditions, in which ultra-high electric and magnetic fields are generated and evolve on ultrafast timescales. Measurement of the radiation emitted from such plasmas provides a powerful diagnostic tool to explore their evolution. For example, $\mathrm{THz}$ radiation emission can be used to diagnose the movement of fast electrons along the target front surface ${ }^{[1-3]}$ and to diagnose the evolution of plasma at the target rear surface $^{[4]}$. In addition, the development of relatively compact high-power laser-driven $\mathrm{THz}$ sources could find applications, for example, in inducing phase transitions in material science ${ }^{[5-7]}$ and for imaging in biology and medicine ${ }^{[8,9]}$.

Correspondence to: Email: paul.mckenna@strath.ac.uk; Y.T. Li, Beijing National Laboratory of Condensed Matter Physics, Institute of Physics, Chinese Academy of Sciences, Beijing, China. Email: ytli@iphy.ac.cn
Previous studies have shown that $\mathrm{THz}$ emission is particularly strong when foil targets are used at the focus of a high-power laser pulse. Strong THz emission is induced at both the front ${ }^{[3]}$ and rear surfaces ${ }^{[4]}$. The sensitivity of $\mathrm{THz}$ generation to laser pulse and interaction parameters (such as the pulse energy, polarization, and incident angle) were reported in both 100-femtosecond-level (or even shorter) duration regimes and in the weakly relativistic or nonrelativistic intensity regimes ${ }^{[3,10-13]}$. The influence of the preplasma generated at the front surface of the target foil has also been explored ${ }^{[10]}$. On increasing the preplasma generation by lowering the laser peak-to-ASE (amplified spontaneous emission) intensity contrast ratio, Li et al. ${ }^{[10]}$ measured reduced $\mathrm{THz}$ emission (a factor of 10 decrease for a similar reduction in contrast ratio, from $3 \times 10^{-8}$ down to $5 \times 10^{-6}$ ). However, working with similar laser pulse parameters to Li et al., Hamster et al. ${ }^{[1]}$ report an opposite trend: a factor of 10 increase in $\mathrm{THz}$ emission when the laser pulse intensity contrast was reduced from $2.5 \times 10^{-6}$ to $5 \times 10^{-4}$. In another experimental study, reported in Sagisaka et al. ${ }^{[2]}$, THz emission was shown to be rather insensitive to the scale length of the preformed plasma. Although three 


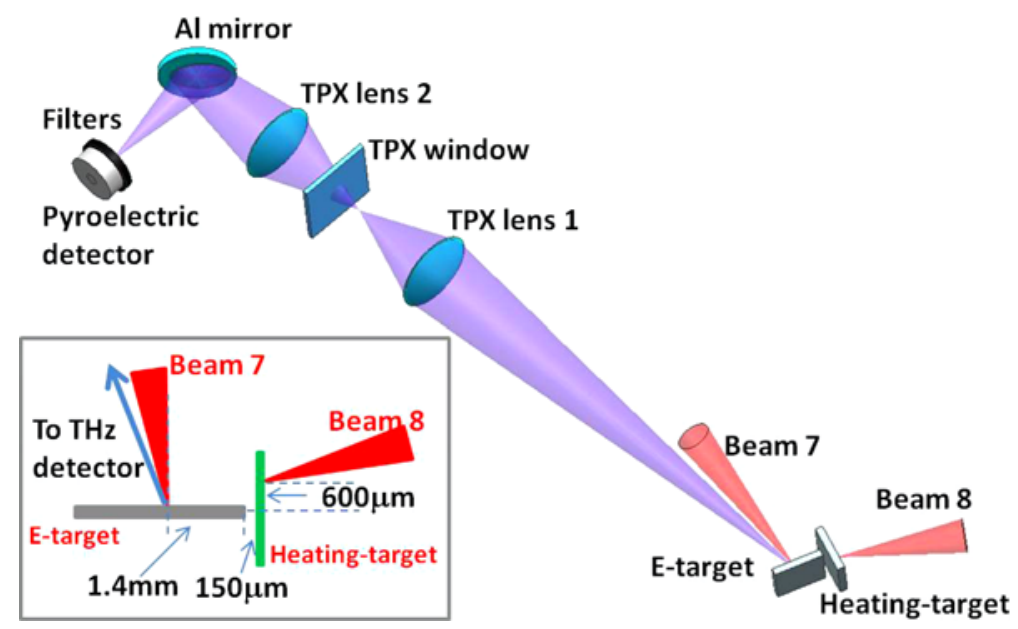

Figure 1. (Color online) Schematic of the experiment layout showing the positions and orientations of the targets, the laser beams, and the THz measurement optics and detector. TPX lens 1, and both targets and beams are in the vacuum sealed by the TPX window. The inset shows a top view of the orientation of the laser beams and targets in closer detail.

different $\mathrm{THz}$ generation mechanisms are invoked to explain the dramatically different observations, further investigation of the influence of preformed plasma is necessary for a fuller understanding of the physics underpinning $\mathrm{THz}$ generation.

In this article, we report on experimental and theoretical investigations on $\mathrm{THz}$ generation from relativistically intense $\left(>7 \times 10^{19} \mathrm{~W} \mathrm{~cm}^{-2}\right)$ laser pulses irradiating solid targets which have been preheated with an intense pulse of multi-MeV protons to induce preplasma formation. A factor of 2 decrease in the total energy of the $\mathrm{THz}$ emission is measured compared to equivalent measurements with an initially cold solid.

\section{Experiment and results}

The experiment was performed using the Vulcan laser at the Rutherford Appleton Laboratory in the UK. Figure 1 shows a schematic of the experimental arrangement. Two picosecond synchronized laser beams, referred to as beams B7 and B8 (beams 1-6 are the long pulse beams of the Vulcan laser facility and are not used in this study), are used to drive $\mathrm{THz}$ generation and proton acceleration for heating, respectively. These beams are hereafter referred to as B7 and B8. B7 delivers pulses of $60 \pm 7 \mathrm{~J}$ on the target with a duration equal to $1 \mathrm{ps}$, resulting in a calculated peak intensity of $(7.6 \pm 0.9) \times 10^{19} \mathrm{~W} \mathrm{~cm}^{-2}$ when focused to a spot of $10 \mu \mathrm{m}$ diameter (FWHM) at an incident angle of $8^{\circ}$ (with respect to the target normal). Note that this relatively small angle of incidence was chosen to minimize the production of transient surface currents of fast electrons ${ }^{[14,15]}$, which can be a dominant driver of $\mathrm{THz}$ generation. B8 pulses have energy and duration equal to $200 \pm 15 \mathrm{~J}$ and $12 \mathrm{ps}$, respectively. They are focused to a $12 \mu \mathrm{m}$ diameter (FWHM) spot, at an incident angle of $15^{\circ}$ (again with respect to the target normal), resulting in a calculated peak intensity of $(1.5 \pm 0.1) \times$
$10^{19} \mathrm{~W} \mathrm{~cm}{ }^{-2}$. Both beams were controlled independently to enable either beam to be used separately or both used together with variable temporal separation of the pulses.

The THz generation target was a $200 \mu \mathrm{m}$ thick amorphous carbon foil ( $3 \mathrm{~mm} \times 3 \mathrm{~mm})$; it is labeled E-target in Figure 1 . The proton source target was a $20 \mu \mathrm{m}$ thick gold foil $(2 \mathrm{~mm} \times 2 \mathrm{~mm})$; it is labeled Heating-target. The inset of Figure 1 shows a top view of the experimental arrangement when both laser beams and targets were employed. The targets were orientated at a slight angle (not illustrated in the figure) such that the expanding proton beam from the Heating-target irradiated the E-target in the region of the B7 focus. The distance between the two laser foci was $1.7 \mathrm{~mm}$. The temporal separation between the pulses was set such that B8 arrived on target $(85 \pm 7)$ ps prior to B7. This enables sufficient time for the propagation of the multi$\mathrm{MeV}$ proton beam from the Heating-target to the E-target, and an additional several tens of picoseconds for preplasma expansion.

The main diagnostic is a broadband pyroelectric $\mathrm{THz}$ radiometer (Model SPI-A-62 from Spectrum Detector Inc.) coupled to a Tektronix oscilloscope. The detector has a flat response coefficient from 0.3 to $21 \mathrm{THz}$, with a calibrated responsivity of $\sim 10^{4} \mathrm{~V} \mathrm{~W}^{-1}$. Two $50 \mathrm{~mm}$ diameter polymethylpentene (TPX) lenses were used to collect and refocus the $\mathrm{THz}$ radiation emitted in the backward direction at $20^{\circ}$ to the B7 laser axis. The focal lengths of these two optics are $f=200 \mathrm{~mm}$ and $f=100 \mathrm{~mm}$. The electromagnetic (EM) radiation was transmitted out of the vacuum chamber through a $2 \mathrm{~mm}$ thick TPX window. The collection angle is limited to $8 \times 10^{-4}$ sr by the aperture size of the second lens. To prevent the detector being irradiated by high-energy particles, the sensor is oriented at an angle to the target and the measurement axis is turned by an aluminium mirror so that only the EM radiation is reflected onto the detector. The 


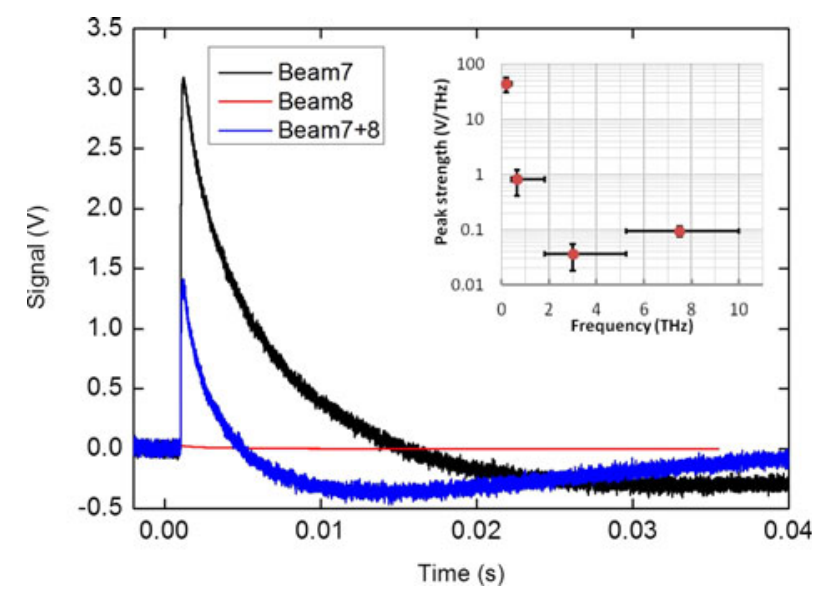

Figure 2. (Color online) Typical THz waveforms (from an oscilloscope) as measured with a pyroelectric detector operating in the range $0.3-1 \mathrm{THz}$. Black: B7 only; red: B8 only; blue: both B7 and B8. The inset shows a reconstructed $\mathrm{THz}$ spectrum from a B7-only shot.

whole detector was housed in a $5 \mathrm{~mm}$ thick aluminum cylindrical cavity, which was further shielded by $50 \mathrm{~mm}$ thick lead bricks, leaving only a $5 \mathrm{~mm}$ diameter entrance hole. Several types of filter were used to ensure that the measured signal was in the $\mathrm{THz}$ frequency regime. A $1 \mathrm{~mm}$ thick piece of high-resistivity silicon (HR-Si) wafer was tightly fixed around the entrance hole to ensure that the arrangement was light-tight. Copper and stainless steel meshes of period $3 \mathrm{~mm}$ and $1 \mathrm{~mm}$ were stacked to remove EM noise below $0.3 \mathrm{THz}$. Different low-pass filters (QMMF mesh filter from QMC Instruments Ltd) were further employed to limit the upper cutoff frequency measured, to $10 \mathrm{THz}, 5 \mathrm{THz}, 1 \mathrm{THz}$, and $0.3 \mathrm{THz}$, enabling a crude $\mathrm{THz}$ spectrum to be determined.

Example waveforms measured with the $\mathrm{THz}$ detector are shown in Figure 2, for three cases: (1) B7 only, (2) B8 only, and (3) both B7 and B8. The filters were chosen to transmit EM radiation in the range $0.3-1 \mathrm{THz}$. For the B7only case, the measured signal peak voltage was $3.2 \mathrm{~V}$ and the duration (FWHM) was $4 \mathrm{~ms}$. The signal decay time is set by the detector response, and is much longer than the actual $\mathrm{THz}$ pulse duration. The pulse energy detected is determined from the calibration to be $0.184 \mu \mathrm{J}$ (equivalent to $230 \mu \mathrm{J} \mathrm{sr}^{-1}$ if assuming isotropic emission and taking account of the diagnostic acceptance solid angle and the attenuations of the lens, window, and filters). The shot-toshot reproducibility of the signal was checked over a series of laser shots on the same target type, and it showed a variation of $<10 \%$. The inset of Figure 2 shows a crude $\mathrm{THz}$ emission spectrum for the B7-only case. The central frequency for each point is the average of adjacent cutoff frequencies of the low-pass edge filters, and the horizontal error bars are obtained from their differentiation. The amplitude of the signal is plotted at the detector voltage per unit frequency (in THz). The vertical error bars reflect the degree of shotto-shot fluctuation and account for systematic errors. The result indicates that the measured $\mathrm{THz}$ emission is mainly at relatively low (up to $1 \mathrm{THz}$ ) frequencies.

To determine if the B8 interaction with the proton source target contributes to the measured $\mathrm{THz}$ signal, measurements were made with B8 only. The energy of the $\mathrm{THz}$ pulse in this case was only $1.4 \mu \mathrm{J} \mathrm{sr}^{-1}$, which is more than two orders of magnitude smaller than the B7 interaction, indicating that the $\mathrm{THz}$ emission is dominated by the $\mathrm{B} 7$ interaction. In shots for which both B7 and B8 are used, i.e., corresponding to proton pre-heating of the main target (E-target), the measured $\mathrm{THz}$ energy decreases, compared to the equivalent case without proton heating, by more than a factor of 2 , to $106 \mu \mathrm{J} \mathrm{sr}^{-1}$.

We now consider the changes to the main target induced by the proton beam. The interaction of B8 with the thin gold target accelerates protons via the target normal sheath acceleration (TNSA) mechanism ${ }^{[16]}$. About $5 \%$ of the laser pulse energy is converted to produce a proton beam with an exponential spectral distribution, with temperature $\sim 2 \mathrm{MeV}$ and cutoff energy equal to $14 \mathrm{MeV}$. The spectrum is measured, using a stack of dosimetry film, with the Etarget in position so that the missing fraction, i.e., the protons absorbed by the E-target, can be determined.

Proton stopping within the target is governed by the Bragg peak energy deposition profile. The resulting target heating and hydrodynamic expansion are modelled using the 1D HELIOS-CR radiation-hydrodynamic code ${ }^{[17]}$ and the PROPACEOS equation of state $(\mathrm{EoS})$. The temporal separation of the arrival of $\mathrm{B} 7$ and $\mathrm{B} 8$ at their respective targets is 85 ps. Protons with energy of $8 \mathrm{MeV}$, for example, will transverse the $1.7 \mathrm{~mm}$ distance from the source to the focal position of B7 in 43 ps, with the larger number of $2 \mathrm{MeV}$ protons arriving after $85 \mathrm{ps}$. The majority of the protons thus arrive and start heating the E-target in the temporal window from around 40 ps prior to the arrival of the peak of B7. This sets the range of expansion times to be modelled in the HELIOS calculations. Note that, as with similar studies involving target heating driven by TNSA protons from a secondary laser-foil interaction (e.g., [18]), we assume that, compared to proton heating, x-ray and fast-electron emission from the secondary foil contributes relatively little to the heating of the front surface of the primary target, due to the Bragg peak energy deposition profile and relatively high directionality of the proton beam. Another factor which must be considered is the amplified spontaneous emission (ASE) of B7 which arrives at the target prior to the protons. Contrast measurements made on the Vulcan laser indicate that the level of the ASE intensity is of the order of $10^{10} \mathrm{~W} \mathrm{~cm}^{-2}$ at $0.1-1 \mathrm{~ns}$, and $10^{11} \mathrm{~W} \mathrm{~cm}^{-2}$ at 50-100 ps prior to the peak of the laser pulse.

\section{Simulations and discussion}

HELIOS simulations were performed for two cases: (1) B7 ASE only (i.e., no proton heating) and (2) B7 ASE plus the 

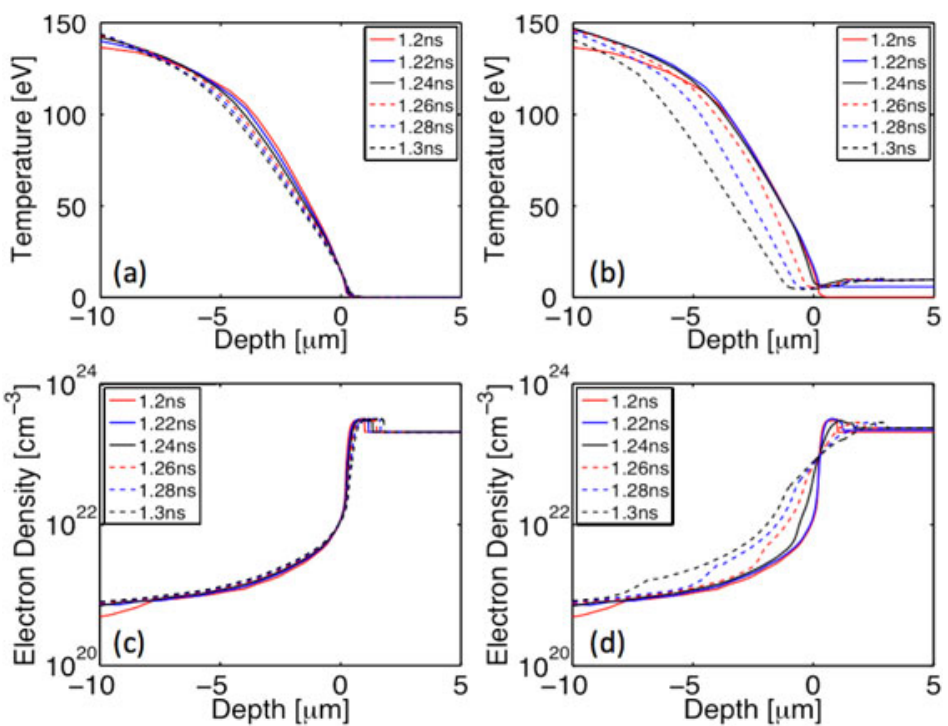

Figure 3. (Color online) HELIOS simulation results. (a) and (c) Temperature and electron density profiles at the front surface of a carbon target at given simulation times, driven by the laser (B7) ASE only. (b) and (d) Corresponding temperature and density profiles for the same target driven by the laser (B7) ASE plus proton heating driven by B8. See the main text for details.

proton beam. The simulation starts at $t=0$ with the arrival of the ASE on target and runs until $t=1.3 \mathrm{~ns}$. The ASE intensity is $10^{10} \mathrm{~W} \mathrm{~cm}^{-2}$ from $t=0-1 \mathrm{~ns}$, and $10^{11} \mathrm{~W} \mathrm{~cm}^{-2}$ from $t=1-1.3 \mathrm{~ns}$. For case (2), $8 \mathrm{MeV}$ protons arrive at the target at $t=1.2 \mathrm{~ns}$, while lower-energy protons arrive later: $1 \mathrm{MeV}$ protons arrive at $t=1.28 \mathrm{~ns}$. The peak of B7 also arrives at $t=1.28 \mathrm{~ns}$.

The resulting target heating and front surface plasma expansion profiles at given simulation times are shown in Figure 3. The ASE-only simulations (Figures 3(a) and 3(c)) show that the primary effect of the ASE pedestal is to heat the underdense region of the target and drive a low-temperature shock wave into the target, resulting in compression of the first micron depth of the target by $\sim 25 \%$. The target heating and expansion driven by the ASE is relatively small. For the case in which the proton beam is added, we find that the first few tens of microns depth from the front surface of the target is heated to temperatures of $\sim 10 \mathrm{eV}$, as shown in Figure 3(b), with an exponential decrease in temperature to about $0.3 \mathrm{eV}$ at a depth of $100 \mu \mathrm{m}$. Similar laser-driven proton heating profiles have been reported in other work ${ }^{[18]}$. Importantly, we find that the target front side expands faster into vacuum in response to this heating, resulting in slightly larger plasma scale length, compared to the case of ASE-only heating.

There have been a few mechanisms proposed for $\mathrm{THz}$ emission from relativistic laser interaction with solid targets. One of them is associated with the space-charge field or laser wakefield excitation at the vacuum-plasma interface driven by the laser ponderomotive force ${ }^{[1,19-21]}$. This mechanism requires a large volume of underdense plasma at densities below $10^{19} \mathrm{~cm}^{-3}$, with scale length longer than the length of the driving laser pulse; otherwise, the generation efficiency is low. Our fluid simulations of preplasma formation reveal that the additional proton heating, which strongly affects $\mathrm{THz}$ emission, mainly changes the density scale length in a localized region at densities of the order of $n_{c}\left(\sim 10^{21} \mathrm{~cm}^{-3}\right)$. It therefore appears that the contribution of $\mathrm{THz}$ emission due to this mechanism is less important. As discussed above, other $\mathrm{THz}$ generation mechanisms are associated with energetic electron production, such as the surface transient current at the front ${ }^{[3]}$ or rear ${ }^{[2]}$, antenna emission ${ }^{[2]}$, and transient radiation ${ }^{[23-25]}$, which occur when the preplasma has moderate-to-short density scale lengths. Generally, all of these mechanisms depend upon the preplasma conditions in two ways, by changing (1) the absorption of laser energy to fast electrons, and (2) the injection properties of the fastelectron distribution (see reference [26] for an example study of the sensitivity to preplasma scale length). A relatively large preplasma usually produces more hot electrons with higher temperature, which often leads to higher transient currents near the target surface when the laser is incident at relatively large angles with respect to the target normal. Two features distinguish the present work from previous studies: the laser (B7) is incident at a relatively small angle $\left(8^{\circ}\right)$, and our approach using proton heating over a large area of the target surface drives quasi-1D preplasma expansion. The results which show that $\mathrm{THz}$ emission is reduced with increasing preplasma expansion, which are in broad agreement with a previous study under different conditions ${ }^{[10]}$, are explained partially by the fact that transient currents formed deeper into the expanded preplasma cannot emit $\mathrm{THz}$ waves out of it because of their low frequency. These effects will be investigated in more detail in further work. 


\section{Conclusion}

In summary, we have compared $\mathrm{THz}$ radiation emission measurements from the interaction of an intense laser pulse with a solid target with and without front surface expansion driven by heating with laser-accelerated protons. The experiment demonstrates that pre-heating and expansion of the target significantly reduces the level of $\mathrm{THz}$ emission, even though the laser is incident at a small angle with respect to the target normal direction. The results suggest that, under these conditions, hot-electron generation and transport effects in the preplasma play an important role in $\mathrm{THz}$ emission.

\section{Acknowledgements}

The authors acknowledge the expert support of the staff at the Central Laser Facility at Rutherford Appleton Laboratory, UK. This work was supported by National Basic Research Program of China (grant nos. 2013CBA01500 and 2014CB339801), National Natural Science Foundation of China (grant nos. 11121504, 11205100, 11220101002 and 11135012), the EPSRC (grant nos. EP/J003832/1 and EP/L001357/1), and the Swedish Research Council.

\section{References}

1. H. Hamster, A. Sullivan, S. Gordon, and R. W. Falcone, Phys. Rev. Lett. 71, 2725 (1993).

2. A. Sagisaka, H. Daido, S. Nashima, S. Orimo, K. Ogura, M. Mori, A. Yogo, J. Ma, I. Daito, A. S. Pirozhkov, S. V. Bulanov, T. Zh. Esirkepov, K. Shimizu, and M. Hosoda, Appl. Phys. B 90, 373 (2008).

3. Y. T. Li, C. Li, M. L. Zhou, W. M. Wang, F. Du, W. J. Ding, X. X. Lin, F. Liu, Z. M. Sheng, X. Y. Peng, L. M. Chen, J. L. Ma, X. Lu, Z. H. Wang, Z. Y. Wei, and J. Zhang, Appl. Phys. Lett. 100, 254101 (2012).

4. A. Gopal, T. May, A. S. Herzer, A. Reinhard, S. Minardi, M. Schubert, U. Dillner, B. Pradarutti, J. Polz, T. Gaumnitz, M. C. Kaluza, O. Jackel, S. Riehemann, W. Ziegler, H. P. Gemuend, H. G. Meyer, and G. G. Paulus, New J. Phys. 14, 083012 (2012).

5. M. K. Liu, H. Y. Hwang, H. Tao, A. C. Strikwerda, K. Fan, G. R. Keiser, A. J. Sternbach, K. G. West, S. Kittiwatanakul, J. Lu, S. A. Wolf, F. G. Omenetto, X. Zhang, K. A. Nelson, and R. D. Averitt, Nature (2012) doi:10.1038/nature11231.

6. M. Chen, X. H. Yuan, and Z. M. Sheng, Appl. Phys. Lett. 101, 161908 (2012).

7. M. Chen, A. Pukhov, X. Y. Peng, and O. Willi, Phys. Rev. E 78, 046406 (2008).

8. B. Ferguson and X. C. Zhang, Nature Mat. 1, 26 (2002).
9. H. T. Chen, W. J. Padilla, J. M. O. Zide, A. C. Gossard, A. J. Taylor, and R. D. Averitt, Nature 444, 597 (2006).

10. C. Li, M. L. Zhou, W. J. Ding, F. Du, F. Liu, Y.-T. Li, W.-M. Wang, Z.-M. Sheng, J.-L. Ma, L.-M. Chen, X. Lu, Q.-L. Dong, Z.-H. Wang, Z. Lou, S.-C. Shi, Z.-Y. Wei, and J. Zhang, Phys. Rev. E 84, 036405 (2011).

11. F. Du, C. Li, M. L. Zhou, W. M. Wang, L. N. Su, Y. Zheng, X. L. Ge, Y. T. Li, J. L. Ma, X. L. Liu, L. Zhang, Z. M. Sheng, L. M. Chen, X. Lu, Q. L. Dong, Z. H. Wang, Z. Y. Wei, and J. Zhang, Sci. China Inf. Sci. 55, 43 (2012).

12. F. Du, C. Li, M. L. Zhou, W. M. Wang, L. N. Su, Y. Zheng, Y. T. Li, J. L. Ma, Z. M. Sheng, L. M. Chen, X. Lu, Z. H. Wang, Z. Y. Wei, and J. Zhang, Sci. China-Phys. Mech. Astron. 55, 589 (2012).

13. Y. Gao, T. Drake, Z. Y. Chen, and M. F. DeCamp, Opt. Lett. 33, 2776 (2008).

14. P. McKenna, D. C. Carroll, R. J. Clarke, R. G. Evans, K. W. D. Ledingham, F. Lindau, O. Lundh, T. McCanny, D. Neely, A. P. L. Robinson, L. Robson, P. T. Simpson, C.-G. Wahlstrom, and M. Zepf, Phys. Rev. Lett. 111, 074802 (2007).

15. R. J. Gray, X. H. Yuan, D. C. Carroll, C. M. Brenner, M. Coury, M. N. Quinn, O. Tresca, B. Zielbauer, B. Aurand, V. Bagnoud, J. Fils, T. Kuhl, X. X. Lin, C. Li, Y. T. Li, M. Roth, D. Neely, and P. McKenna, Appl. Phys. Lett. 99, 171502 (2011).

16. S. C. Wilks, A. B. Langdon, T. E. Cowan, M. Roth, M. Singh, S. Hatchett, M. H. Key, D. Pennington, A. MacKinnon, and R. A. Snavely, Phys. Plasmas 8, 542 (2001).

17. J. J. MacFarlane, I. E. Golovkin, and P. R. Woodruff, J. Quant. Spectrosc. Radiat. 99, 381 (2006).

18. A. Mancic, J. Robiche, P. Antici, P. Audebert, C. Blancard, P. Combis, F. Dorchies, G. Faussurier, S. Fourmaux, M. Harmand, R. Kodama, L. Lancia, S. Mazevet, M. Nakatsutsumi, O. Peyrusse, V. Recoules, P. Renaudin, R. Shepherd, and J. Fuchs, High Energy Density Physics 6, 21 (2010).

19. Z. M. Sheng, H. C. Wu, K. Li, and J. Zhang, Phys. Rev. E 69, 025401(R) (2004).

20. Z. M. Sheng, K. Mima, J. Zhang, and H. Sanuki, Phys. Rev. Lett. 94, 095003 (2005).

21. L. M. Gorbunov and A. A. Frolov, J. Exp. and Theoret. Phys. 102, 894 (2006).

22. A. Gopal, S. Herzer, A. Schmidt, P. Singh, A. Reinhard, W. Ziegler, D. Brommel, A. Karmakar, P. Gibbon, U. Dillner, T. May, H.-G. Meyer, and G. G. Paulus, Phys. Rev. Lett. 111, 074802 (2013).

23. J. Zheng, K. A. Tanaka, T. Miyakoshi, Y. Kitagawa, R. Kodama, T. Kurahashi, and T. Yamanaka, Phys. Plasmas 10, 2994 (2003).

24. C. B. Schroeder, E. Esarey, J. van Tilborg, and W. P. Leemans, Phys. Rev. E 69, 016501 (2004).

25. W. J. Ding, Z. M. Sheng, and W. S. Koh, Appl. Phys. Lett. 103, 204107 (2013).

26. P. McKenna, D. C. Carroll, O. Lundh, F. Nurnberg, K. Markey, S. Bandyopadhyay, D. Batani, R. G. Evans, R. Jafer, S. Kar, D. Neely, D. Pepler, M. N. Quinn, R. Redaelli, M. Roth, C.-G. Wahlstrom, X. H. Yuan, and M. Zepf, Laser Part. Beams 26, 591 (2008). 